\title{
Application of oxygen A-band equivalent width to disambiguate downwelling radiances for cloud optical depth measurement
}

\author{
Edward R. Niple ${ }^{1}$, Herman E. Scott ${ }^{1}$, John A. Conant ${ }^{1}$, Stephen H. Jones ${ }^{1}$, Frank J. Iannarilli ${ }^{1}$, and \\ Wellesley E. Pereira ${ }^{2}$ \\ ${ }^{1}$ Aerodyne Research, Inc., Billerica, MA, USA \\ ${ }^{2}$ Air Force Research Lab, Albuquerque, NM, USA \\ Correspondence to: Frank J. Iannarilli (franki@aerodyne.com)
}

Received: 4 March 2016 - Published in Atmos. Meas. Tech. Discuss.: 8 March 2016

Revised: 5 July 2016 - Accepted: 12 August 2016 - Published: 31 August 2016

\begin{abstract}
This paper presents the three-waveband spectrally agile technique (TWST) for measuring cloud optical depth (COD). TWST is a portable field-proven sensor and retrieval method offering a unique combination of fast $(1 \mathrm{~Hz})$ cloudresolving $\left(0.5^{\circ}\right.$ field of view) real-time-reported COD measurements. It entails ground-based measurement of visible and near-infrared (VNIR) zenith spectral radiances much like the Aerosol Robotic Network (AERONET) cloud-mode sensors. What is novel in our approach is that we employ absorption in the oxygen A-band as a means of resolving the COD ambiguity inherent in using up-looking spectral radiances. We describe the TWST sensor and algorithm, and assess their merits by comparison to AERONET cloud-mode measurements collected during the US Department of Energy's Atmospheric Radiation Measurements (ARM) TwoColumn Aerosol Project (TCAP). Spectral radiance agreement was better than $1 \%$, while a linear fit of COD yielded a slope of 0.905 (TWST reporting higher COD) and offset of -2.1 .
\end{abstract}

\section{Introduction}

\subsection{Motivations and contribution}

Accurate global climate model (GCM) predictions are absolutely essential. They are needed not only to help mitigate damage from unwanted climate changes but also to determine what, if any, interventions can reverse those changes directly. Yet clouds, because of their complexity and inherently random nature, remain a primary challenge to GCM accuracy. Direct optical measurements of cloud optical depth (COD) serve a number of atmospheric and climate change science and monitoring purposes. In fundamental cloud process and observational studies, the column-integrated nature of COD imposes a strong calibration constraint on measurements by vertical-profiling instruments such as lidar and cloud radar (Kikuchi et al., 2006). Localized COD measurements serve as concise validation checks on cloud radiative transfer (RT) models. These purposes are often costeffectively served by ground-based COD instruments. Globally distributed routine time series observations of COD are similarly useful in GCM validation. Although such observation grids are routinely available from satellite retrievals from short-wavelength reflectances (Nakajima and King, 1990), there remains substantial added value from ground-based COD instrument networks, such as the Aerosol Robotic Network (AERONET) (in cloud mode). This added value is ascribed to the continuing need for independent validation of satellite-retrieved COD (Liu et al., 2013), since satellite sensor calibrations degrade in orbit and suffer their own measurement biases.

A further use that we have made for ground-based COD measurements is providing ground truth for field experiments of optical contrast propagation through clouds. In these applications we have placed emphasis on (1) fast measurement rate, e.g., $1 \mathrm{~Hz}$; (2) cloud-resolving narrow field of view (FOV) applicable to scattered clouds as opposed to overcastonly situations; and (3) real-time reporting. As this combination of features was to our knowledge unavailable from existing instruments (circa 2010), we developed the TWST sensor and its COD retrieval algorithm. 
COD and cloud droplet effective radius $r_{\mathrm{e}}$ together are the minimal required parameters to determine a liquid water path (LWP) and thus a connection between cloud macroobservables and microphysical parameters. To retrieve COD and $r_{\mathrm{e}}$ from short-wavelength radiances, a longstanding approach (Nakajima and King, 1990) has been to employ two wavelengths, one at a non-absorbing (e.g., visible/NIR) and the other at an absorbing wavelength for liquid water, e.g., longer than $1500 \mathrm{~nm}$. Indeed, the AERONET cloud mode has recently adopted a longer-wavelength channel for this purpose (Chiu et al., 2012), and other ground-based optical sensors employ a sufficiently broad spectral range (McBride et al., 2011; Liu et al., 2013; Fielding et al., 2014). For the TWST sensor's present state of development, we purposely chose not to operate beyond $1100 \mathrm{~nm}$ due to cost and complexity burdens we wished to avoid. As the primary data product of TWST is COD, in Sect. 3.2.2 we provide evidence of retrieved COD's relative insensitivity to $r_{\mathrm{e}}$ for low to moderate COD values.

Our TWST technical approach was inspired by the development of AERONET cloud mode, circa 2010 (prior to its employment of the $1640 \mathrm{~nm}$ channel), which we summarize next.

\subsection{Prior art of COD retrieval from zenith spectral radiance}

The use of zenith visible and near-infrared (VNIR) spectral radiances to measure COD advanced with the work of Marshak et al. (2004) using a technique first suggested by Marshak et al. (2000) and Barker and Marshak (2001). In order to resolve the COD ambiguity, i.e., the lack of a bijective relationship between spectral radiance and COD, they use spectral radiances at red $(670 \mathrm{~nm})$ and NIR $(870 \mathrm{~nm})$ wavelengths which sit on opposite sides of the chlorophyll red edge feature of the albedo of vegetated terrain. The technique was validated at the Atmospheric Radiation Measurements (ARM) Southern Great Plains (SGP) site in Oklahoma by comparison to more conventional techniques (Microwave Radiometer and Multifilter Rotating Shadowband Radiometer). In the work of Chiu et al. (2006) the preliminary validation was extended. In Chiu et al. (2010) the technique was improved by switching to blue $(440 \mathrm{~nm})$ and NIR $(870 \mathrm{~nm})$ wavelengths. Furthermore, it was applied to selected AERONET sensors during what otherwise were sunobscured sleep periods and has since become known as the AERONET cloud mode.

\subsection{TWST synopsis and paper outline}

The TWST sensor is a zenith-staring narrow-field-of-view (NFOV) VNIR spectral radiometer built around an inexpensive commercial compact grating spectrometer (CGS) with a nominal $2.5 \mathrm{~nm}$ resolution. The technological sophistication and robustness of the TWST instrument derives almost en- tirely from its commercial components; we neither depend upon nor make any remarkable claims about sensor design or suitability. In Sect. 2, we describe the TWST sensor and its field-worthiness; present example data; and discuss its calibration, including dark-current correction. In Sect. 4.2.1, within our Measurements section, we establish TWST radiometric veracity and stability by comparison to coincident AERONET spectral radiance observations over a period of several weeks.

Although we customarily record the full spectral record spanning about $350-1000 \mathrm{~nm}$, the TWST COD retrieval presently makes use of a sparse set of spectral bins decomposed into three spectral factors: the spectral radiances at 440 and $870 \mathrm{~nm}$ (SR440 and SR870) and the equivalent width (EQW) of the oxygen A-band centered near $760 \mathrm{~nm}$. TWST is not using A-Band spectrometry to retrieve a numerical COD. Like AERONET cloud mode, TWST employs model-generated lookup tables of spectral radiance to COD. In particular, TWST tables relate SR440 to COD. TWST differs from cloud mode in its resolution of the aforementioned COD ambiguity. Cloud mode (circa 2010) employs a twodimensional ordinate space involving the sum and difference of SR440 and SR870, which is bijective to pairing of COD and effective cloud fraction. TWST instead first determines the cloud optical thickness regime, thin or thick, and thus whether to reference the thin or thick branch of the SR440 to COD lookup table. The novelty of TWST is its determination of thickness regime from a multivariate temporal filter employing a color index (the SR440/SR870 ratio) and the slope of the plot of SR440 vs. A-Band EQW. TWST development status presently circumscribes it to the regime of low-moderate-altitude water clouds and small-moderate solar zenith angles (SZAs). Defining TWST's precise operational boundaries is a future task.

In Sect. 3.1 we discuss the TWST COD retrieval, including its physical basis, COD error sensitivity to primary uncertainties, implementation details, and an explanatory example of the retrieval technique operating on a time sequence of sensor data. In Sect. 4 , in addition to the aforementioned spectral radiance comparison, we establish TWST COD retrieval accuracy by comparing to coincident AERONET cloud-mode COD. We present and discuss the quality of agreement in both an illustrative several-hour time series and the cumulative correlation over a several-week period of the TwoColumn Aerosol Project (TCAP) field campaign.

\section{The TWST sensor}

\subsection{Design and characteristics}

The heart of the TWST sensor is a zenith-pointing calibrated spectroradiometer. We elected to design the sensor around a commercial CGS, given the significant advances in miniaturization, rugged monolithic construction, and linear array de- 
tectors. Several advantages accrue from this design choice, the most important to our COD measurement application being the acquisition of spectral radiances at high signal-tonoise ratio (SNR) and high temporal resolution, attributed to the multiplex advantage provided by the CGS.

The key specifications for the TWST COD sensor are listed in Table 1. Here we are excluding extreme ambient conditions outside the range of -10 to $+40{ }^{\circ} \mathrm{C}$ that require special temperature control. The spectral resolution defined by the spectrometer configuration is currently $\sim 2.5 \mathrm{~nm}$ (including convolution with slit function), and the sampling interval is $\sim 0.3 \mathrm{~nm}$. With integral order-sorting filter, the stray light level is cited to be $<0.1 \%$. The temporal resolution determined by the available sunlight, spectrometer throughput, and linear focal plane array (FPA) detector characteristics - is a variable sampling interval from $1 \mathrm{~ms}$ to $60 \mathrm{~s}(1 \mathrm{~s}$ typical). A typical TWST spectrum recorded at $1 \mathrm{~s}$ interval consists of 400 co-added snapshots each of $2.5 \mathrm{~ms}$ integration time. The SNR for a single snapshot is limited to $400: 1$ due to photo-electron noise based on the electron well depth of 160000 . When readout noise is included, this drops to $275: 1$. With 400 co-adds, the $1 \mathrm{~s}$ maximum-signal SNR is therefore about $5500: 1$.

We have now built and tested a few different configurations of the TWST sensor, but each includes the basic elements represented in the schematic design in Fig. 1; a companion photograph looking inside a recent model is shown in Fig. 2. The entrance window (A) is slanted to shed rain drops. A simple mechanical shutter $(\mathrm{S})$ for recording dark spectra, selected for its reliability and effective light blocking performance, is located just inside the input window and driven by an inexpensive stepping motor. An incoming light baffle (B) limits the total field of view to $0.5^{\circ}$ FWHM. A collecting lens $(\mathrm{C})$ then focuses the filtered light onto the end of a $400 \mu \mathrm{m}$ diameter optical fiber (D) which feeds the light into the CGS (E). The entire system is contained in an IP66 (NEMA 4X) rated sealed enclosure with desiccant to prevent water condensation over deployment periods of several months. Our design has proven to be field-worthy, easily transportable, and stable over a wide range of environmental conditions as supported in Sect. 4.2.1. We have experienced no instances of condensation inside the sealed TWST enclosure while operating in humidity and temperature conditions well below the dew point.

\subsection{Example spectral data}

The TWST retrieval algorithm uses three spectral factors (Fig. 3): the spectral radiances at 440 and $870 \mathrm{~nm}$ (SR440 and SR870) and the EQW of the oxygen A-band (Sect. 3.3.1). Figure 3 shows example calibrated spectral measurements for nearly identical SZA, but for clear sky and a range of COD values in the thin optical thickness regime. The overall radiance level as well as the depth of the oxygen A-band absorption is observed to increase with COD.

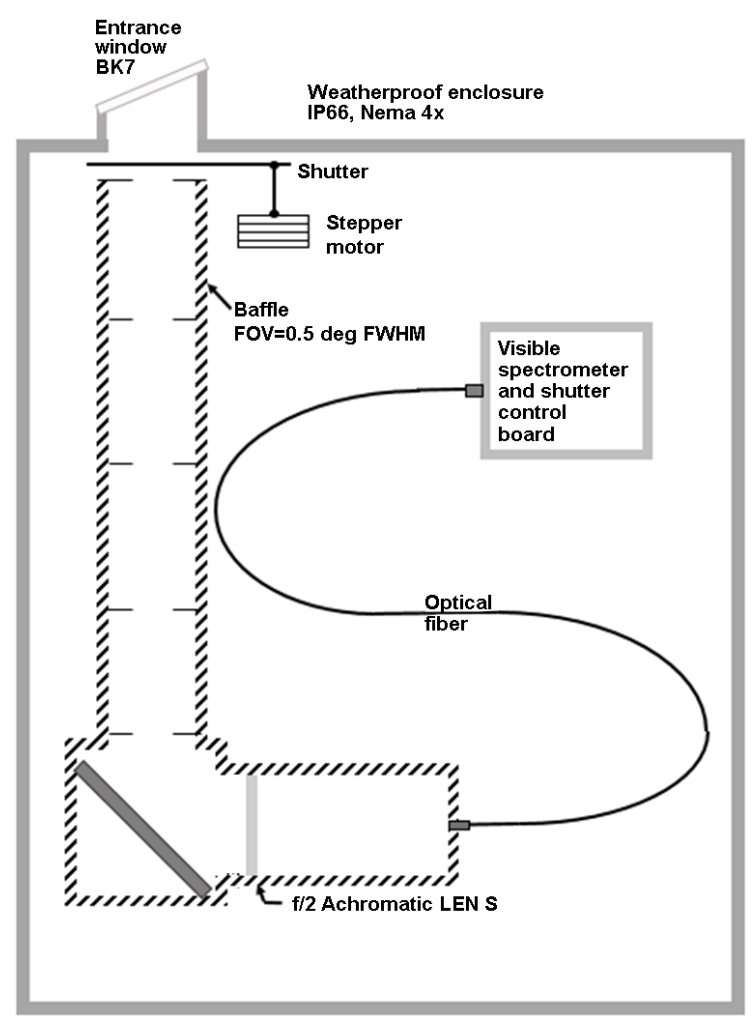

Figure 1. Simplified schematic of the TWST cloud optical depth sensor.

\subsection{Calibration and dark-current correction}

There are two forms of calibration that must be managed for any technique that uses spectroradiometers: wavelength and radiometric. The wavelength calibration of the compact grating spectrometers used in our TWST sensors has proven stable over periods of months. Furthermore, the TWST approach does not rely on resolving spectral line structure. The 440 and $870 \mathrm{~nm}$ spectral radiance levels, due to their shallow spectral slopes (Fig. 3), and A-Band EQW value, due to its accumulation over many spectral bins, are relatively insensitive to foreseeable thermal shifting of the spectral sampling grid.

TWST spectral radiance calibration is performed at the beginning and end of every field deployment and more frequently as needed. Our calibration source is a Labsphere Uniform Radiance Standard integrating sphere. It is well known that the standard incandescent source lamps age and need to be replaced periodically. Like other long-time users, we find these lamps to be the largest source of uncertainty and absolute error in our radiometric calibration procedure; that uncertainty is $\sim 5 \%$. During each calibration we set the integration period, number of snapshot co-adds, and aperture radiance to span the range of field conditions anticipated for sunlit clouds, and then we derive a linear photoresponsivity coefficient in the usual manner. These radiometric calibra- 
Table 1. TWST cloud optical depth sensor specifications.

\begin{tabular}{ll}
\hline & $\begin{array}{c}\text { TWST COD sensor specifications } \\
\text { for ambient temperature range }-10 \text { to }+40{ }^{\circ} \mathrm{C}\end{array}$ \\
\hline Weight & $20 \mathrm{lbs}$ \\
Power and communication for optical head & $5 \mathrm{Vdc},<250 \mathrm{~mA}$ via a single USB 2.0 cable connection to computer for \\
power and data & $11 \times 8 \times 8$ in. plus 12 in external sun baffle, or $13 \times 10 \times 6$ in. with \\
Size & internal sun baffle \\
& Blue sky to COD 100 \\
Operating range & 0.001 for optically thin clouds \\
COD sensitivity & IP66, NEMA 4 X sealed enclosure with desiccant \\
Weatherproof environmental container & $1 \mathrm{~Hz}$ (typical), variable sampling interval from 0.1 to $60 \mathrm{~s}$ \\
Data-logging rate & $0.5^{\circ}$ \\
Field of view & $350-1000 \mathrm{~nm}, \sim 2.5 \mathrm{~nm}$ \\
Spectral range, resolution & 440,760, and $870 \mathrm{~nm}$ \\
Spectral bands currently used in COD retrieval &
\end{tabular}

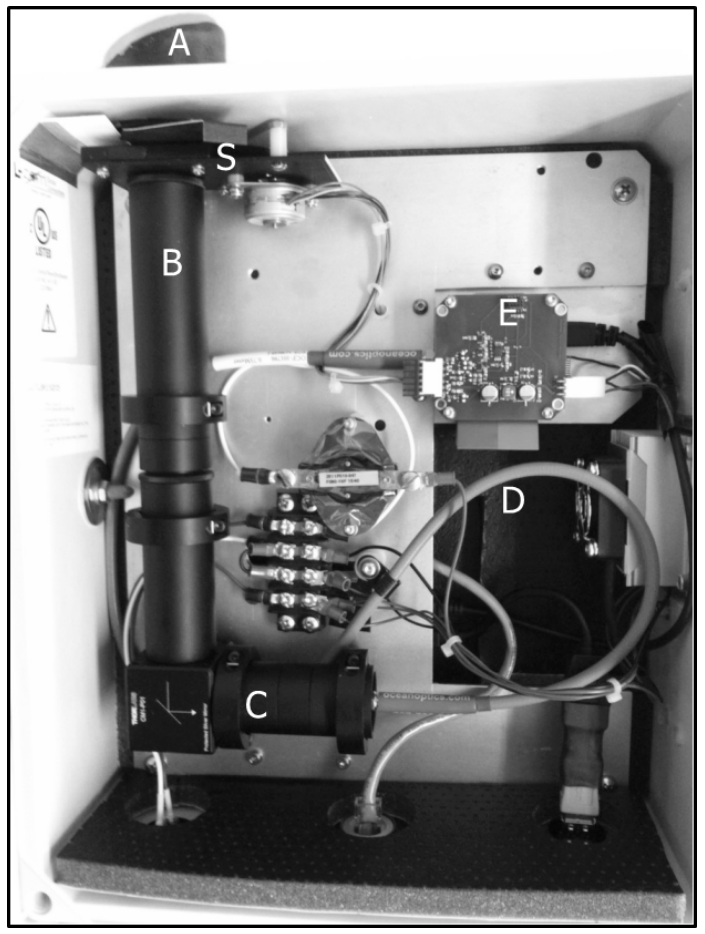

Figure 2. A view inside the TWST cloud optical depth sensor. See text for labeled component descriptions.

tion records for each TWST unit are kept and compared over periods of years to monitor the stability of each unit for its lifetime. Having records for some units over 2-4 years, we find changes in the calibration of 1-3\%, well within the uncertainty of our calibration lamps, which as noted above is on the order of $5 \%$.

The spectrometer's silicon CCD detector outputs are susceptible to offset drift, typically driven by changes in ambient temperature, but the detector array contains light-shielded dark-reference detectors intended to automatically track and

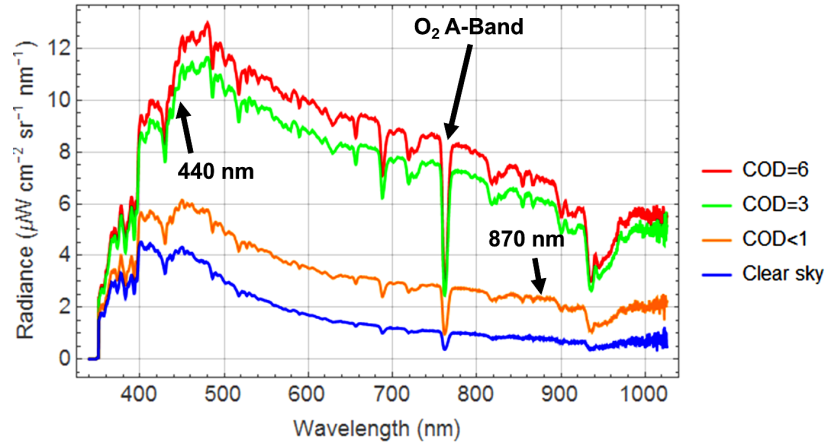

Figure 3. Example spectra measured by TWST sensor, delineating the three spectral factors currently used in TWST retrieval algorithm. Spectra measured at $\mathrm{SZA}=65^{\circ}$ within $1 \mathrm{~min}$.

subtract such drift. In addition, TWST employs a mechanical shutter for frequent collection of dark spectra, typically a $1 \mathrm{~s}$ dark spectrum every $60 \mathrm{~s}$. The dark correction (offset subtraction) applied to each recorded spectrum is spline-extrapolated from earlier collected dark spectra. In Sect. 4.2.1, the effectiveness of these calibration methods is evaluated by comparison to coincident AERONET spectral radiance observations over a period of several weeks.

\section{TWST cloud optical depth retrieval method}

\subsection{Section outline}

The TWST retrieval algorithm employs model-generated lookup tables to convert zenith spectral radiance at $440 \mathrm{~nm}$ (SR440) to a numerical value of COD. However, the TWST algorithm first determines the cloud optical thickness regime, thin or thick, and thus whether to reference the thin or thick branch of the SR440 to COD lookup table. We first discuss the somewhat conventional spectral radiance to numerical 
COD lookup, including table generation, COD error sensitivity to principal uncertainties via radiative transfer simulations, and technique of interpolation between table entries. Then we discuss the determination of the optical thickness regime. This entails discussion of why the oxygen A-band and its EQW metric are informative of the thickness regime. We introduce the "nose" plot of SR440 vs. EQW, and its generic slope characteristics are revealed as a key to resolving the thin-thick ambiguity; the algorithm does not use model-generated nose plots. We explain the need and basis for the SR440/SR870 ratio as color index. The color index and nose plot slope metrics are combined in a multivariate temporal filter that continually updates the estimate of optical thickness regime. To illustrate its operation and how it copes with 3-D cloud effects, we discuss an example nose plot time sequence and filtered results.

\subsection{Numerical spectral radiance to COD lookup}

\subsubsection{Radiative transfer construct}

Assuming the sensor's FOV does not include the Sun, the zenith spectral radiance consists of solar radiation scattered by the molecules, aerosols, and cloud water droplets in the FOV, which may include radiation that has been scattered multiple times from the atmosphere and the terrain. The VNIR spectral band (Fig. 3), at a moderate spectral resolution of $2 \mathrm{~nm}$, shows a broad baseline with multiple narrow absorption features. Many of these are due to water vapor, as well as Fraunhofer lines. The spectral radiance at $440 \mathrm{~nm}$ is in a region relatively free from atmospheric gaseous absorption and is thus suitable as a COD proxy. We chose $440 \mathrm{~nm}$ for TWST radiance-to-COD lookup because that is a wavelength used by AERONET cloud-mode sensors, which serve as a source for comparative validation.

The model used for generating $440 \mathrm{~nm}$ radiance-to-COD lookup tables is the MODerate resolution atmospheric TRANsmission (MODTRAN) atmospheric radiative transfer code (Berk et al., 2006). MODTRAN5 incorporates the Discrete Ordinates Radiative Transfer (DISORT) code (Stamnes et al., 1988) for plane-parallel stratified media, i.e., idealized one-dimensional radiative transfer (1DRT). Calculations are done for a typical water stratus cloud above a stated ground albedo, for a stated nominal aerosol profile, over a grid of COD and SZAs. Figure 4 is a graphical depiction of sample tables. For any SZA, there is a "bright-point" radiance where the idealized 1DRT cloud radiance reaches a maximum, typically occurring for a COD between 2 and 8, as seen in Fig. 4 . Real clouds manifest three-dimensional radiative transfer (3DRT) effects, including radiances exceeding the idealized 1DRT bright-point radiances (Marshak et al., 2000). When faced with such exceedances, the TWST retrieval algorithm reports the COD corresponding to the bright-point radiance but flags an out-of-bounds ("3-D cloud") condition.

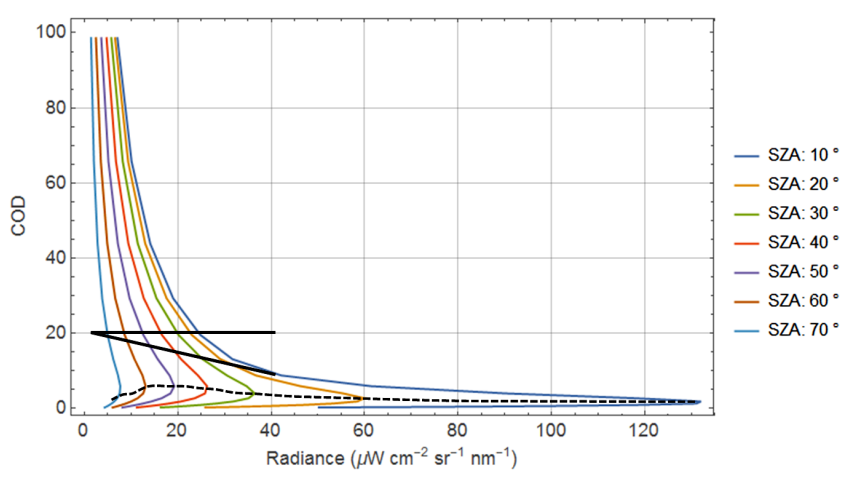

Figure 4. $440 \mathrm{~nm}$ radiance to COD lookup tables for various SZAs. The radiance peak for each curve is its 1DRT "bright-point" radiance. The black solid and dotted line markers are referred to in various sections of the text.

\subsubsection{COD error sensitivity to radiative transfer parameter uncertainties}

The TWST algorithm currently operates without any information on the droplet size distribution or the cloud base height, and with a prior estimate of the ground albedo and aerosol loading profile. We do not consider deviation of the actual from nominal aerosol profile, as such perturbation from the baseline aerosol optical depth (AOD) is typically a small contributor to reported COD. We performed some initial sensitivity studies on these remaining parameters. The albedo sensitivity findings below will prove of value in helping to explain the minor disagreement bias between coincident TWST and AERONET COD observations (Sect. 4.2.2).

Because our implementation of the TWST algorithm uses a radiance database generated with the MODTRAN model, we studied $440 \mathrm{~nm}$ radiances from four different cloud types parameterized within MODTRAN, which assume Mie scattering, lognormal droplet size distribution, and liquid water refractive index. These types have effective radii of 12.0 (cumulus), 7.2 (altostratus), 8.3 (stratus), and $6.7 \mu \mathrm{m}$ (stratocumulus). Water cloud drop-size distributions typically vary from an effective radius of 1-20 $\mu \mathrm{m}$ (see, e.g., Chiu et al., 2006). We modeled clouds with fixed base height of $0.5 \mathrm{~km}$ and fixed physical thickness of $0.5 \mathrm{~km}$. For each cloud type we varied the LWP enough to achieve $550 \mathrm{~nm}$ CODs between 0 and 100; LWP was used because it is an input to MODTRAN. COD values were estimated from LWP using the Wood and Hartmann (2006) modification to the Stephens (1978) formula as described in Chiu et al. (2012). Figure 5 shows the computed $440 \mathrm{~nm}$ vertical radiances plotted vs. LWP and COD. The different size distributions lead to different radiances for the same LWP as expected. However, in the COD vs. radiance plot the curves overlay closely, at least for $\mathrm{COD} \leq 20$. We verified this intuitive result using a simple standalone two-stream computation as an alternative to the DISORT algorithm included within MODTRAN 


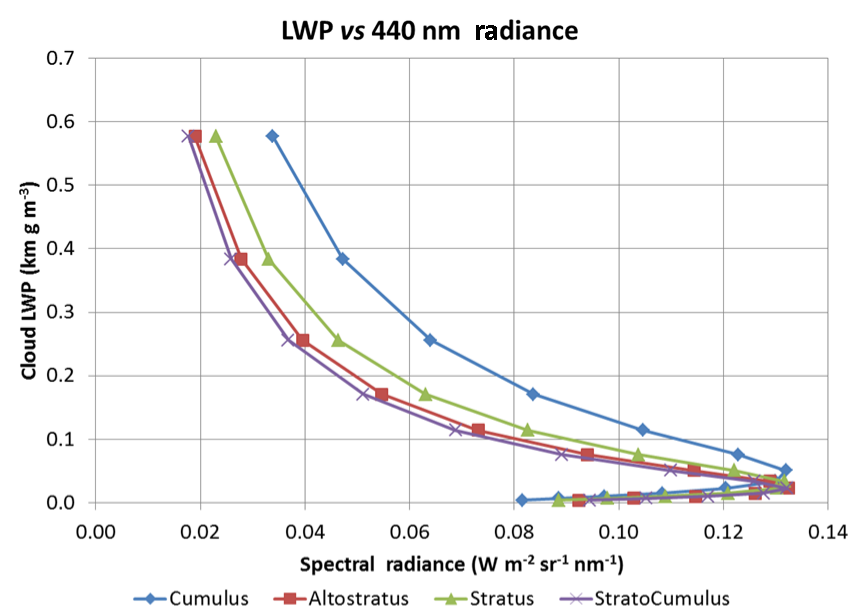

(a) Cloud LWP vs. radiance

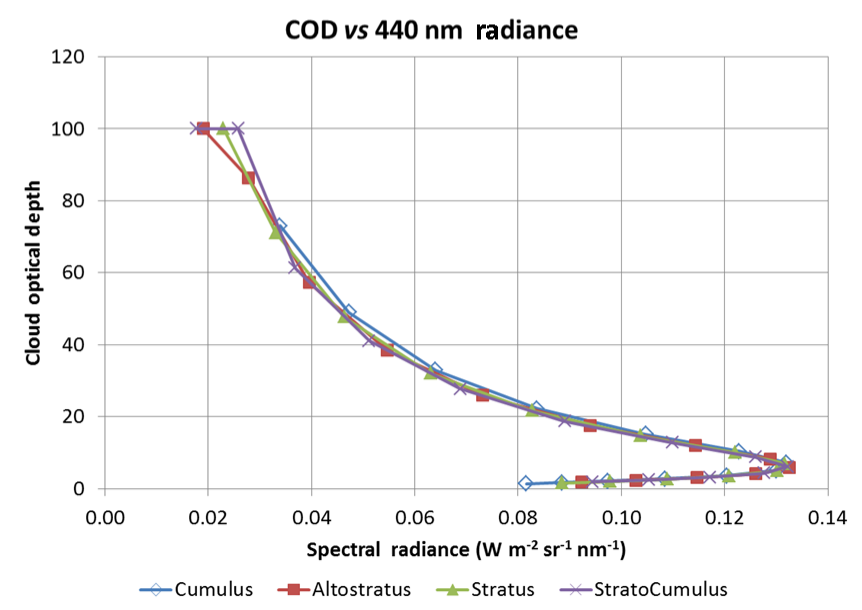

(b) Cloud OD vs. radiance

Figure 5. Relationship between spectral radiance at $440 \mathrm{~nm}$ wavelength and (a) liquid water path (LWP) and (b) cloud optical depth, for four different cloud types: effective radii of 12.0 (cumulus), 7.2 (altostratus), 8.3 (stratus), and $6.7 \mu \mathrm{m}$ (stratocumulus).

(Stamnes et al., 1988). These results are corroborated by the more extensive sensitivity results of McBride et al. (2011). This relative insensitivity of COD with effective radius gives us some confidence in reporting COD values in the face of the variety of water clouds.

Inter-reflections between the ground and a thick cloud can be significant unless the Earth albedo is low. At the $440 \mathrm{~nm}$ lookup table wavelength most Earth cover types have albedo of 0.2 or less as shown in Fig. 6 with samples of the Advanced Spaceborne Thermal Emission and Reflection Radiometer (ASTER) database (Baldridge et al., 2009); notable exceptions are for white sand, fresh snow, and ocean ice. To characterize the sensitivity of retrieved COD due to albedo uncertainty, we used MODTRAN to compute $440 \mathrm{~nm}$ zenith radiances for a low-altitude stratus cloud for albedos of 0.0 ,

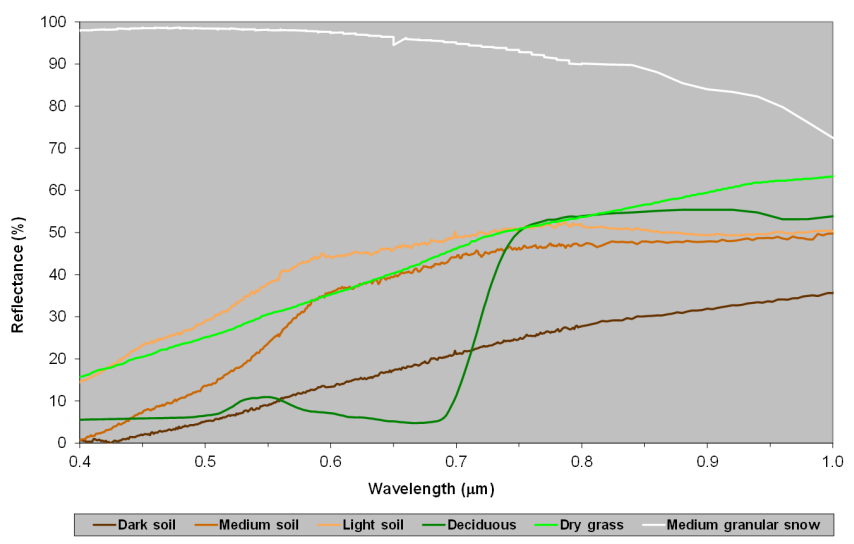

Figure 6. Spectral albedos of common Earth cover types from the ASTER database.

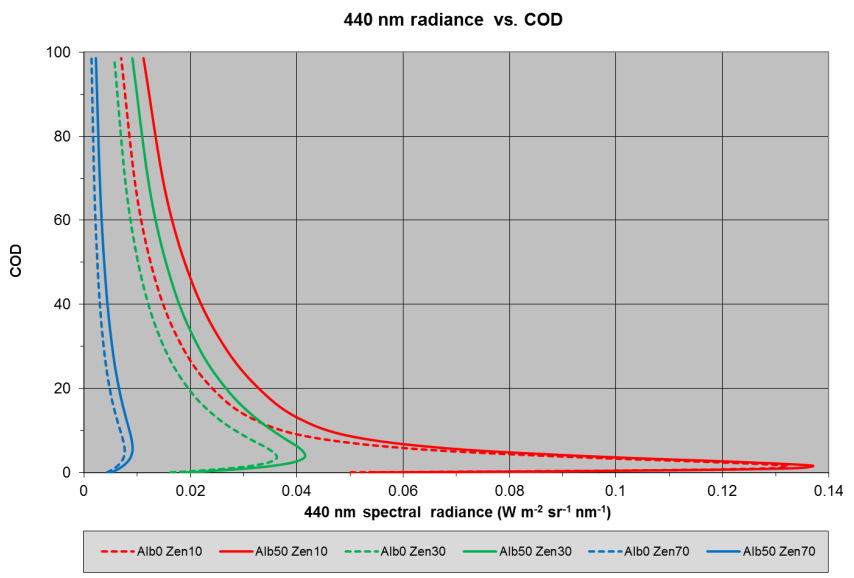

Figure 7. COD vs. $440 \mathrm{~nm}$ spectral radiance for solar zenith angles of 10,30 , and $70^{\circ}$ and for ground albedos of 0 and $50 \%$.

$0.1,0.2$, and 0.5 , over a range of solar zeniths and cloud optical thicknesses. Figure 7 presents a first-order indication of sensitivity and plots $440 \mathrm{~nm}$ radiances vs. COD for albedo bounds of 0.0 and 0.5 . The thin-thick ambiguity, the strong variation with solar zenith, and the weaker variation with ground albedo are evident in these plotted results.

The plot in Fig. 8a further explores this sensitivity and shows the signed change in retrieved COD value for an unexpected increase in the ground albedo from 0.1 (for which the radiance-to-COD lookup tables are computed) to 0.2. Each curve, for either thick or thin cloud, pertains to some fixed percentage of the aforementioned 1DRT bright-point radiance "Lbrt" (which varies with SZA; cf. Fig. 4). These curves show that a higher-than-expected albedo implies retrieval of a lower (higher) COD in the thick (thin) regime. The largest change we found was $\triangle \mathrm{COD}$ of 5 , but that occurred for very thick clouds, such that the relative change was only $10 \%$. Near the bright point (red curves in Fig. 8a) the COD vs. radiance is quite nonlinear (Fig. 4), and thus the red curves, 


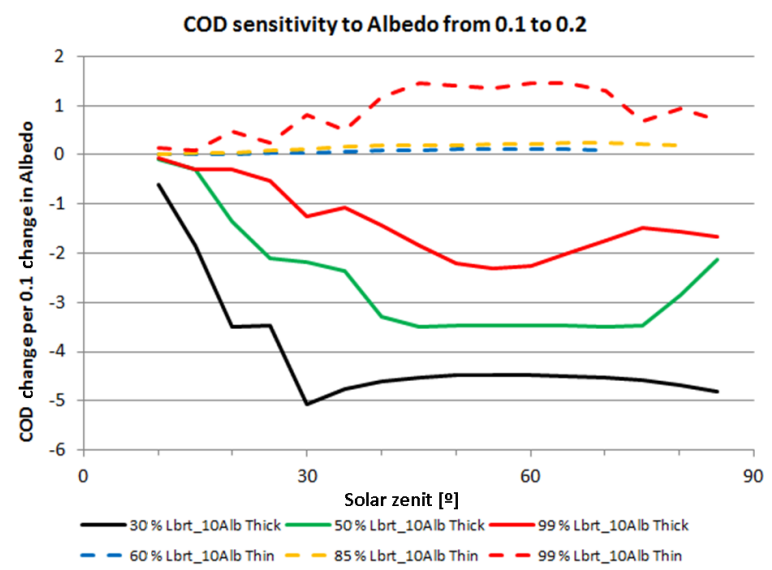

(a) Retrieved COD sensitivity: MODTRAN

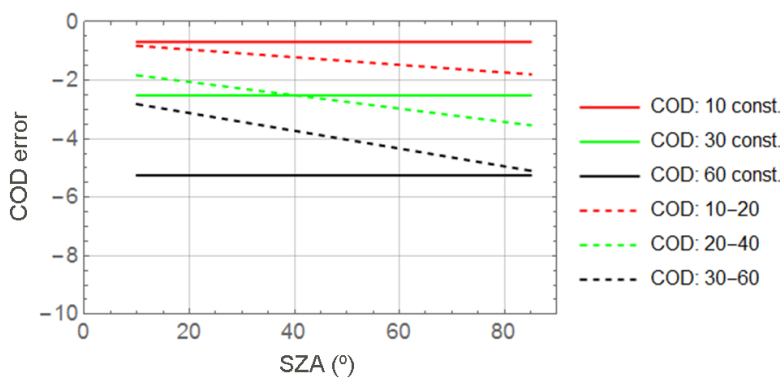

(b) Retrieved COD sensitivity: Asymptotic RT

Figure 8. Retrieved COD sensitivity to change in albedo from 0.1 to 0.2 (a) for different relative cloud radiance levels as computed using MODTRAN and (b) as computed using asymptotic RT relations. See text for details.

approximated by linear interpolations for this study, are less precise and jagged.

To provide analytic support to these albedo sensitivity findings, we performed calculations employing asymptotic radiative transfer (ART) theory relations as elucidated by King (1987) and Melnikova et al. (2000). Both MODTRAN and our ART calculations compute the $440 \mathrm{~nm}$ radiance and optical thickness for cloud with phase function asymmetry parameter $g=0.86$ and single-scattering albedo $\omega_{0}>0.9999997$ (i.e., conservative scattering). ARTcomputed sensitivities are plotted in Fig. 8b, and compare well in both trend and magnitude against the thick regime curves of Fig. 8a, to which ART theory pertains (here COD > 9). These ART sensitivities are processed from the more directly obtained ART calculations plotted in Fig. 9. The connection between Figs. 9 and $8 \mathrm{~b}$ is depicted by the dotted path shown in Fig. 9. The reader is directed to graphically determine the error in retrieved COD value by first starting with a given true COD value, tracing rightward from that $y$ intercept parallel to the $x$ axis, and reaching a curve pair for a given SZA. The right curve of the pair registers the actual radiance measured for the unexpected 0.2 albedo, but the left

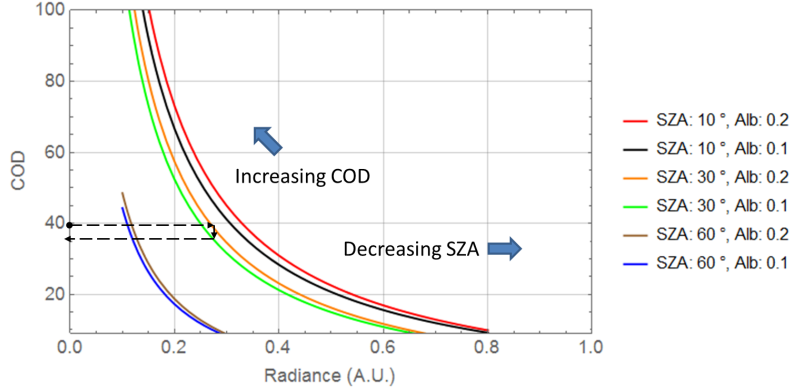

Figure 9. Retrieved COD sensitivity to change in ground albedo from 0.1 (left curves) to 0.2 (right curves) for three different SZA curve pairs, computed using asymptotic RT relations. See text for details.

curve is radiance-to-COD lookup table computed for the expected 0.1 albedo. So at the intersection with the right curve, the reader traces down parallel to the $y$ axis to intercept the left curve and then traces leftward back to the $y$ axis and reads out the lower COD value. The solid curves of Fig. $8 \mathrm{~b}$ are not exactly comparable to those of Fig. 8a in that the former are for constant COD whereas the latter are for constant relative radiance (with respect to the 1DRT bright-point radiance). To corroborate that the COD error in fact decreases with SZA, the dashed curves of Fig. 8b better (but not exactly) correspond to constant relative radiance. These dashed curves have COD decrease with decreasing SZA, which referring to Fig. 4 yields a more stationary relative brightness (downward-sloping line marker) than for COD constant with SZA (horizontal line marker).

At this point it is important to note that the spectral radiance chosen at some other wavelength than $440 \mathrm{~nm}$ could be used for the radiance-to-COD lookup. In principle the choice of wavelength depends upon freedom from atmospheric gaseous absorption and on the ground albedo of the measurement site. One wants a wavelength with the lowest absolute albedo uncertainty to minimize errors in the COD due to errors in the assumed albedo for the MODTRAN5 computations. This flexibility in the choice of wavelength is basis of the term "spectrally agile" within the TWST acronym.

For low-altitude water clouds, uncertainty in the cloud base height has a negligible impact on COD retrieval. We ran MODTRAN for cloud base heights of $500 \mathrm{~m}$ and $2 \mathrm{~km}$, iterating over $10 \mathrm{COD}$ and 11 solar zenith angles for each. The $440 \mathrm{~nm}$ radiances were nearly identical, as shown in the scatterplot of Fig. 10.

\subsubsection{Lookup table interpolation}

Various lookup table algorithms were investigated to reach a reasonable tradeoff between accuracy and speed. The MODTRAN5 tables are preprocessed as follows. Referring to Fig. 4, for each $\mathrm{SZA}_{i}$ entry, a cubic spline curve 


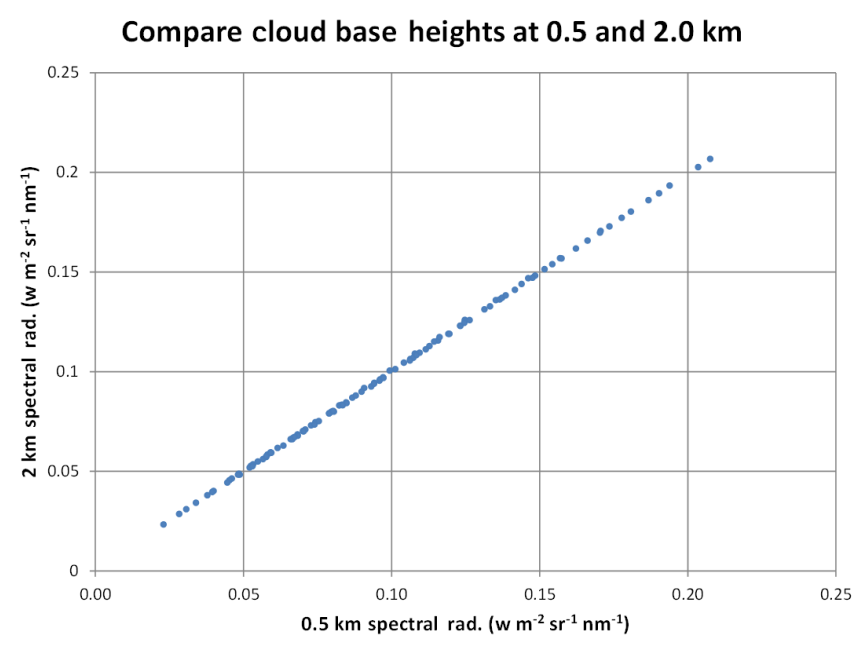

Figure 10. Scatterplot comparing $440 \mathrm{~nm}$ radiance computed for cloud base heights of $500 \mathrm{~m}$ and $2 \mathrm{~km}$, each varying over $10 \mathrm{COD}$ and 11 solar zenith angles.

SR440(COD; $\mathrm{SZA}_{i}$ ) is fit to its SR440-vs-COD table for both optical thickness regimes. A bright-point radiance vs. SZA spline curve Lbrt(SZA), depicted by the dotted black curve in Fig. 4, is fit through the bright-point radiances across the $\mathrm{SZA}_{i}$ entries. During operation, the algorithm identifies the tabulated $\mathrm{SZA}_{j}$ closest to the current solar zenith angle $\mathrm{SZA}_{\text {obs. }}$. Then a working copy of its spline curve $\mathrm{SR} 440\left(\mathrm{COD} ; \mathrm{SZA}_{j}\right)$ is linearly scaled in radiance so that its bright point matches $\operatorname{Lbrt}\left(\mathrm{SZA}_{\mathrm{obs}}\right)$. This scaled curve is then used to look up the COD value for the measured SR440.

\subsection{Optical thickness regime determination}

\subsubsection{Cue from oxygen A-band equivalent width}

The well-known oxygen A-band centered near $760 \mathrm{~nm}$ (Mulliken, 1928; Wark and Mercer, 1965) has been used for many years to study the atmosphere from satellite and ground-based sensors. Pfeilsticker et al. (1998) first used well-resolved A-band spectra to study the probability density function of geometrical path lengths for skylight transmitted from clear and cloudy skies to the ground, following the suggestion of Pfeilsticker et al. (1996) and Harrison and Min (1997). The A-band is virtually free from absorption by other atmospheric constituents (Pfeilsticker et al., 1998) except for aerosol and cloud continua extinction plus a very small amount of line absorption by water vapor (Fig. 11). Thus its continua-normalized (Sect. 3.3.2) spectral-average quantity, termed the equivalent width (EQW), provides a direct measurement of the average amount of oxygen-densityweighted photon path length from the Sun to the sensor. Since oxygen is uniformly mixed in the atmosphere, this is related to the photons' physical path lengths. Therefore the EQW supplies useful information about whether a zenith ra-

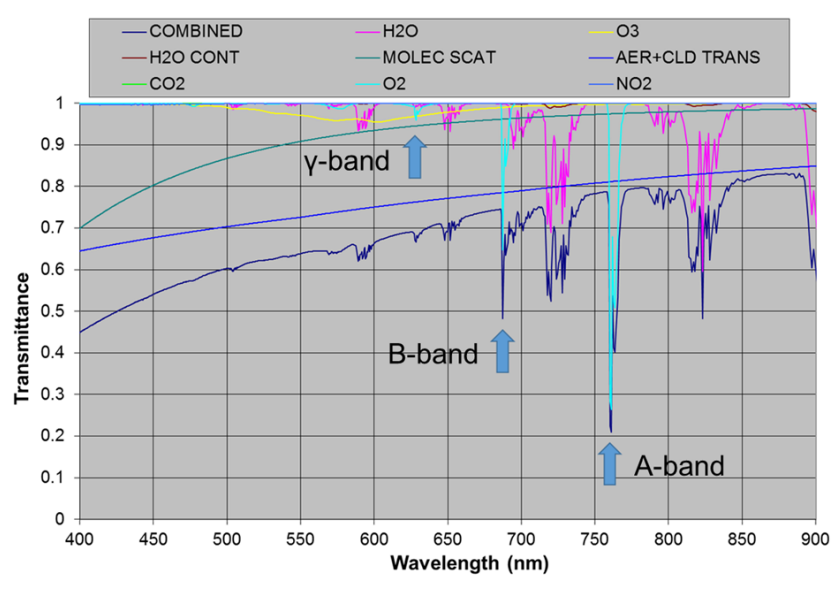

Figure 11. MODTRAN5 calculation of atmospheric transmittance for a ground-based zenith path to space. The oxygen A-Band is virtually free of absorption by any other species except for aerosol and cloud continua extinction. At $0.1 \mathrm{~cm}^{-1}$ spectral resolution, $\mathrm{H}_{2} \mathrm{O}$ (water vapor) has a minimum transmittance of 0.9972 across the A-band.

diance measurement is in the optically thin or optically thick regime. A virtue of EQW is that it may be stably computed from low-resolution spectral data such as from the TWST sensor, as detailed in Sect. 3.3.2.

Of course, other factors cause EQW to change besides COD. Changes in the SZA produce decreases in EQW with time during the morning and increases in the afternoon. Changes in the density-weighted average cloud thickness and cloud altitude also affect the EQW independent of the COD.

\subsubsection{Calculation of EQW}

The equivalent width is computed from the spectral radiances between 750 and $785 \mathrm{~nm}$ by fitting a straight line to the continuum baseline from 750 to 760 and from 770 to $785 \mathrm{~nm}$ (Fig. 11), then dividing each measured spectral radiance by the corresponding linear fit baseline to produce a transmittance value, and then summing these values across the absorption band. This calculation normalizes away the continuum transmittance. The veracity of these calculations depends on accurate spectrometer dark-current calibration and subtraction (discussed in Sect. 2.3). Otherwise, a dark bias of the spectral radiance would falsely alter the computed transmittances and EQW value.

\subsection{3 "Nose" plot of SR440 vs. EQW}

COD is a two-valued function of up-looking spectral radiance, while oxygen equivalent width is a monotonic function of COD for COD $\sim>1$. By plotting SR440 vs. EQW as COD increases from no cloud to thick clouds, one traces out a "nose-like" shape (Fig. 12). For the very lowest COD values, EQW decreases with increasing $\mathrm{COD}$ and the slope is negative. Beyond about $\mathrm{COD}=1$, the lower portion of the 


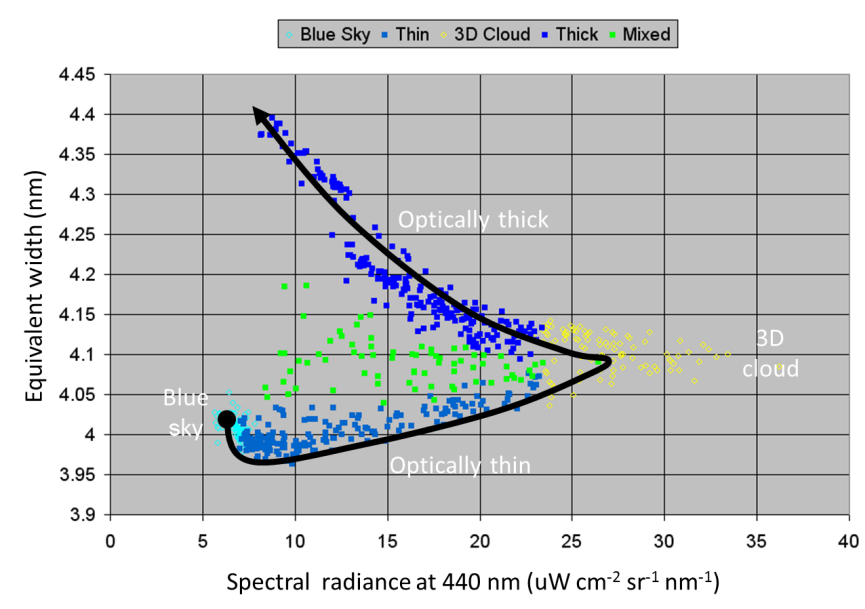

Figure 12. The "nose" plot of SR440 vs. EQW, indicating a trajectory with increasing COD. The data sample categories (colors) are described in the text.

nose where the slope is positive corresponds to the optically thin regime; the upper portion where the slope is negative corresponds to the optically thick regime. Within a span of several seconds, passing clouds most often do not trace out a complete nose but only a small segment of it as the cloud evolves and drifts in the wind over the sensor. Notwithstanding the lowest COD values discussed further below, whether the cloud changes involve increases or decreases of the COD, the slope of the corresponding segment indicates the cloud's optical thickness regime. This is the TWST basis for resolving the COD ambiguity.

The nose plot in Fig. 12 includes a smooth curve, based on MODTRAN5 computations but elastically stretched to fit the depicted data points over a 4 min measurement where the COD varied strongly between the indicated blue-sky, thin, and thick regimes, and points deviating well away from the ideal 1DRT smooth curve. These deviating points are classified as either 3-D cloud based on their SR440 exceedance of the 1DRT bright-point radiance value (Sect. 3.2.1) or as "mixed" points attributed to heterogeneous cloud structure within the field of view, itself a 3DRT effect. The classification of the remaining data points into optically thin, thick, or blue-sky regimes was corroborated against coincident all-sky camera video. Although the MODTRAN5 computed nose plot curve supports these regime classifications, it is important to note that the TWST algorithm does not employ model-generated nose plot curves to guide its thickness regime determination. Indeed, the particular shape and slope of a computed nose plot curve varies, as it should, with the unknown physical cloud thickness. Instead, the algorithm exploits the aforementioned positive slope (thin) and negative slope (thick) generic properties of the nose plot.

\subsubsection{Thickness regime filter}

The cloud optical thickness regime determination operates in two distinct radiance domains. When the COD is very low, e.g., $\mathrm{COD} \leq 1$, the amount of radiation in the NIR is very small and the SNR of the EQW is low (e.g., "clear sky" in Fig. 3), and thus the nose plot slope SNR is too low to resolve the thickness regime. The ratio of SR440 to SR870, termed the color index, has a much higher SNR and is more reliable in this regime. This, of course, is a simple consequence of the wavelength dependence of Rayleigh scattering. For low-moderate-altitude water clouds, small-moderate SZA, and typical $440 \mathrm{~nm}$ ground albedos less than 0.2 (Fig. 6), our data analyses have found a hard threshold of 4 to be a sure indicator of optically very thin clouds (e.g., clear sky in Fig. 3) and a soft threshold of $2<$ index $<4$ to be a strong indicator (e.g., "COD $<1$ " in Fig. 3). When the color index is less than 2 , the cloud's optical thickness is not well correlated with the index, and the algorithm must rely on the nose plot slope.

Figure 13 re-depicts the nose plot data points of Fig. 12, this time connecting a subset of points with line segments to indicate adjacent samples in a 2 min time series. If measured nose plots followed an idealized 1DRT curve as indicated in Fig. 12, the determination of thickness regime would be nearly trivial. A linear regression over a short time segment would suffice. Clearly more complex logic is required, yet it is visually evident that local coherence could be exploited. The qualitative reduction in ambiguity afforded by examining a sufficient time record suggests the use of a filter with memory. For example, for passing or evolving clouds spatially well-resolved within a narrow field of view, the thickness regime should not switch rapidly between thick and thin except possibly near the bright point (thick-thin regime boundary) where a switch is inconsequential to the retrieved COD. We implemented a time-varying hysteresis filter to effectively avoid this unwanted switching. The hysteresis action is achieved by keeping track of the maximum and minimum values of equivalent width over a predetermined time interval, typically about $3 \mathrm{~min}$. In order to drive the filter toward a different thickness regime, the hysteresis limits must be exceeded. The output of the hysteresis filter is discrete ternary: $-1,0$, or 1 , corresponding to thick, indeterminate, or thin. Finally, this ternary variable is input to a linear, single-pole autoregressive (AR(1)) filter to afford additional smoothing. The output of this filter is thresholded and used as the thickness regime estimate.

\subsubsection{Example operation of thickness regime filter}

Figure 13 includes time series plots of SR440 and algorithm retrieved COD corresponding to the connected data points in the nose plot. The dark- and medium-blue points indicate thick and thin cloud, respectively, while light-cyan points indicate clear sky. Yellow points are for SR440 values greater than the 1DRT bright-point value and are indicative 

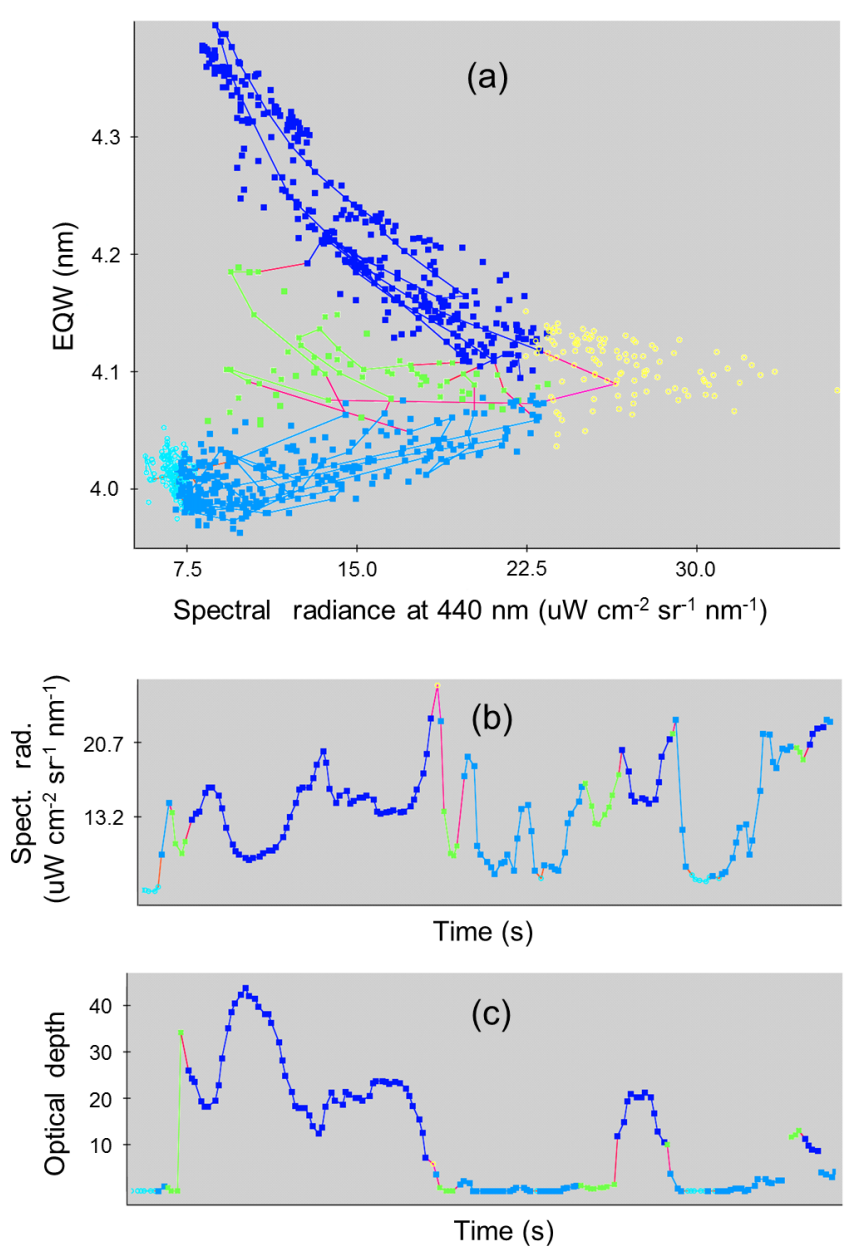

Figure 13. (a) Nose plot of data taken over several minutes. The colors are described in the text. Simultaneous time plots of (b) spectral radiance at $440 \mathrm{~nm}$ and (c) retrieved optical depth corresponding to the nose plot (a).

of 3-D cloud effects. The green aforementioned mixed points (Sect. 3.3.3) were determined manually and are those where the values deviated strongly from the overall nose curve locus; red line segments serve to indicate where along the time series those deviations start and end. Using an instrument with small field of view and fast time response, we expect to see good temporal coherence in the data, and in fact the radiance time plot shows that the cloud optical thickness regime does not change randomly. One can see that the green-labeled points are always transitions between thin and thick cloud which did not follow the idealized nose curve through the bright point (at a radiance of about 23). The EQW values of those points are reasonable, but the radiances are lower than expected. Our supposition at this time is that some of these are due to further 3-D cloud structure effects, but leading to darker radiances rather than the bright radiances of the yellow points, while others points may be due to spatial averaging over the field of view.

\section{Measurements and comparisons to AERONET}

\subsection{The Two-Column Aerosol Project}

TCAP was a 1-year measurement campaign directed by the ARM division of the US Department of Energy. It was designed to quantify aerosol properties, radiation, and cloud characteristics, producing a database to assist climate modeling studies. The ground-based campaign involved the ARM Mobile Facility (AMF) suite of sensors deployed at the ARM Highlands in Cape Cod, Massachusetts. The aerial campaign involved two aircraft loaded with remote and in situ sensors. Measurements were performed from July 2012 until June 2013.

With the kind permission and assistance of the TCAP project, the TWST sensor was set up on Cape Cod near the AERONET cloud-mode sensor and Total Sky Imager (TSI), which are part of AMF, on 17 May 2013. Data were collected continuously for a period of 6 days. Some minor adjustments were then made to the sensor configuration, and then data were collected for the next 30 days until 27 June, when the AMF was taken down in preparation for its next deployment. During this period about 50000 spectra were collected by TWST every day at $1 \mathrm{~s}$ intervals during the day.

\subsection{AERONET cloud-mode and TWST data comparison}

During the time TWST was deployed on Cape Cod, AERONET collected 266 COD values that overlapped TWST measurements. In addition, 8609 overlapping spectral radiance values at $440 \mathrm{~nm}$ were collected. Since all TWST's COD values were based on SR440 measurements, it is important to compare the SR440 values before comparing the COD values.

\subsubsection{Spectral radiance comparison}

This required careful time synchronization between the AERONET and TWST data times. A linear drift of $0.27 \mathrm{~s} \mathrm{day}^{-1}$ was determined by least squares fits to the individual days with a $4 \mathrm{~s}$ difference between the high gain $(\times 8)$ A and low gain $(\times 1) \mathrm{K}$ measurements from AERONET. The result (Fig. 14) shows that both sensors were reporting SR440 values in very good agreement. The rms difference was $0.63\left(\mu \mathrm{W} \mathrm{cm}{ }^{-2} \mathrm{sr}^{-1} \mathrm{~nm}^{-1}\right)$. A simple linear fit without a constant yielded a slope of 1.003 (0.0004). TWST values at high spectral radiance showed some evidence of nonlinear response.

Several conclusions follow from the very good agreement among TWST and AERONET spectral radiances. The first is the expectation of a COD comparison not influenced by TWST spectral radiance errors. As a corollary, the COD comparison should not be unduly influenced by different fields of view $\left(1.2^{\circ}\right.$ for AERONET vs. $0.5^{\circ}$ for TWST) and zenith pointing (robotic control for AERONET vs. fixed tri- 


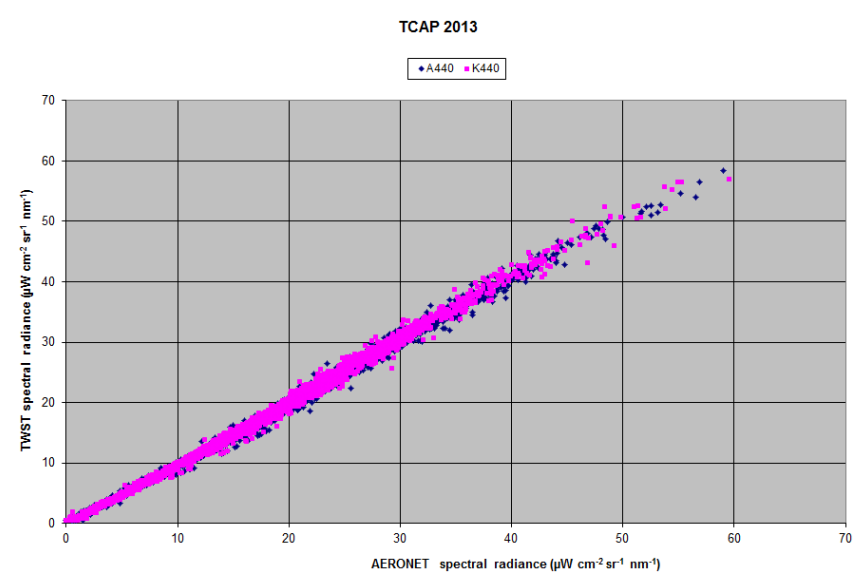

Figure 14. Comparison of TWST and AERONET cloud-mode spectral radiances at $440 \mathrm{~nm}$ wavelength.

pod with bubble level for TWST), given the close agreement over many different cloud conditions. The sensors were laterally displaced by about $3 \mathrm{~m}$, and for a $1 \mathrm{~km}$ cloud base altitude their field-of-view footprints are 20 and $8 \mathrm{~m}$. Of course, the agreement only proves consistency, not accuracy, for either sensor. The second is the radiometric stability of TWST during its TCAP deployment. This is corroborated by the stability of the four pre- and one post-test radiometric calibrations, with the photoresponsivity coefficient at $440 \mathrm{~nm}$ for 9 July being $98.1 \%$ of that for 17 May.

\subsubsection{COD comparison}

A comparison of COD values between TWST and AERONET cloud-mode must recognize the time-sampling differences between them. For these comparisons only the $90 \mathrm{~s}$ average COD was available for AERONET cloud mode, which is a form of trimmed mean based on up to 10 instantaneous COD measurements during each measurement period (see Chiu et al., 2010; Sect. 2.3).

A time series comparison of COD is shown in Fig. 15. The agreement indicates that the TWST thickness regime filter is able to track the rapidly changing COD. The ensemble comparison of the COD values (Fig. 16) shows evidence of the two different types of errors in the TWST and AERONET cloud-mode algorithms: errors in cloud thickness regime and errors in numerical COD. To attempt a comparison, each plot data point represents the average of the 90 instantaneous COD measurements produced by TWST during that same $90 \mathrm{~s}$ period for AERONET.

Of the 244 overlapping COD values, 235 (96\%) showed the same cloud thickness regime. Some analysis was done in an effort to determine whether TWST or AERONET cloud mode was probably correct. For the nine cases where AERONET and TWST disagreed on the thickness regime, detailed nose plots were generated to see if we could visually extract more than the simple slope information used in the

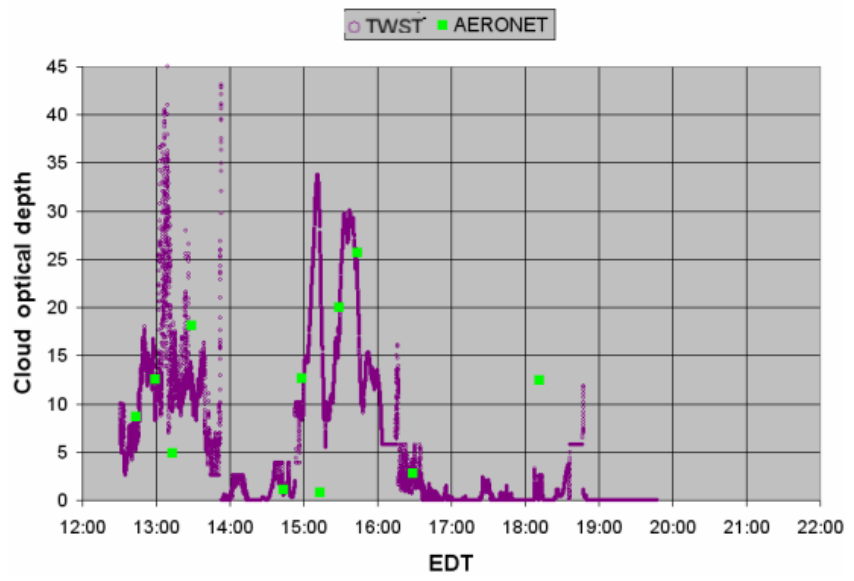

Figure 15. COD time series comparison between AERONET and TWST for 14 June 2013 at the ARM TCAP field campaign.

automated algorithm. Four of the cases produced close to the ideal nose shape, indicating that the TWST thickness regime was probably correct. For the other five cases, the nose plot was too distorted to determine the thickness regime, indicating that the TWST thickness regime was probably incorrect and should have been assigned the "unknown" label.

A linear fit of TWST to AERONET cloud-mode COD, for the 235 cases of thickness regime agreement, for a fixed zero intercept, found a slope of 0.843 (TWST reporting higher COD values) with an rms difference of COD 3.2. This was repeated while dropping the two high COD value outlier points (Fig. 16), but the slope only changed by $1 \%$. No evidence of a constant offset between TWST and AERONET cloud mode was found. However, the sparsity of such evidence is due to the relatively few optically thin COD cases available from AERONET, due to the secondary mission status of its cloud mode. (When skies are largely clear, AERONET executes its primary mission of aerosol optical depth and microphysical property retrieval measurements.) Therefore, another linear fit, this time with a free intercept, found a slope of 0.905 and constant offset of -2.1 .

The two primary candidates for causing the observed disagreements are differences in the TWST and AERONET cloud-mode lookup tables and effects from the trimmed mean process. There may also be some residual effects due to FOV and pointing differences, although these are not expected to be large due to the very good spectral radiance agreement (Sect. 4.2.1). A partial explanation centering on the lookup tables is the difference in assumed ground albedos between the sensors. The TWST SR440-to-COD lookup table generated from MODTRAN used a weighted average of water, deciduous vegetation, dead pine, and sand albedos, resulting in an Earth albedo at $440 \mathrm{~nm}$ of 0.078545 . On the other hand, AERONET updates its ground albedo episodically every few days from MODIS data products or a (rolling) 16-day average MODIS historical database (Chiu 


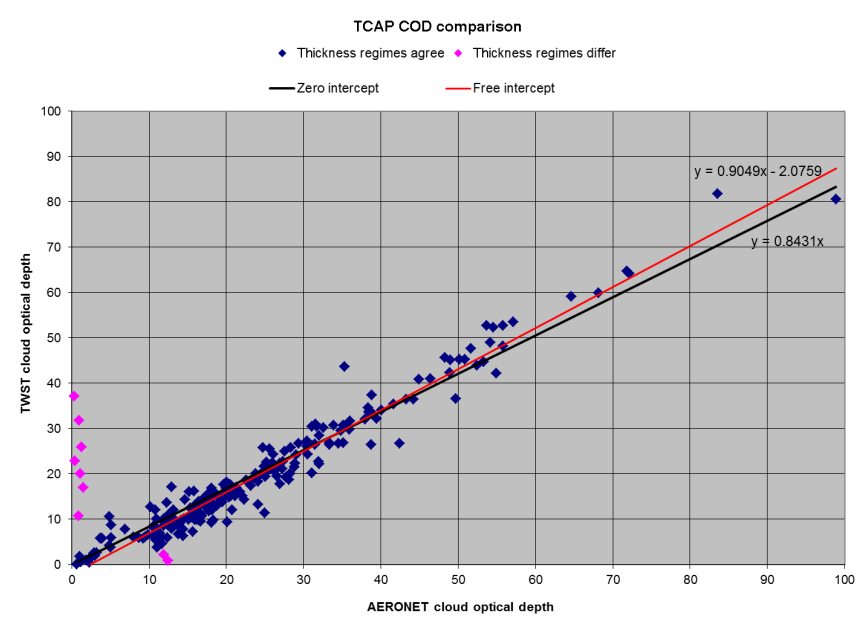

Figure 16. Comparison of TWST and AERONET cloud-mode cloud optical depths.

et al., 2012). For this dataset, the AERONET-employed albedos were lower than that assumed for TWST, varying between 0.02 and 0.04 , with an average of 0.03 . Most of the sample points are in the optically thick regime, and according to our albedo sensitivity discussion (Sect. 3.2.2), a lowerthan-expected albedo implies TWST retrieval of higher COD values in the thick regime, consistent with the linear fits. Figure 8 depicts an approximately constant relative COD retrieval error of about $10 \%$ per 0.1 albedo increment. The 0.05 average difference in assumed albedo therefore explains about half $(0.05)$ of the difference between a slope of unity and the fitted slope (0.905).

It must always be kept in mind that the COD values determined by TWST and AERONET cloud mode are only equivalent 1DRT quantities. Violations of 1-D assumptions are present in nearly all our measurements to some extent. This includes (1) cases where the observed spectral radiance is greater than that possible for any 1-D cloud, (2) cases where deviations from the ideal nose plot are too high for any 1-D cloud, and (3) the many cases where the rapid variation in spectral radiance is too high for 1-D clouds.

\section{Conclusions}

Overall the good agreement between TWST and AERONET cloud-mode cloud optical depth values, across many weeks of coincident field deployment, validates the performance of the three-waveband spectrally agile technique as well as the field-worthiness of the TWST sensor. Although the spectrally agile nature of TWST was not investigated in this study, its advantage over fixed spectral bands for cases with high albedo at $440 \mathrm{~nm}$ may be the focus of a future study. Although our error sensitivity studies in Sect. 3.2.2 and the agreement with AERONET over many weeks of the TCAP campaign lend confidence in applying TWST for nominal conditions, future efforts will ascertain and, where possible, extend the operational limits (e.g., SZA, ground albedo) of the TWST retrieval algorithm.

One of the most notable results of our experience with TWST is the high signal-to-noise ratio available in the high temporal $(1 \mathrm{~s})$, spatial $\left(0.5^{\circ}\right.$ field of view $)$, and spectral $(2.5 \mathrm{~nm})$ resolution data TWST generates. At peak signal, at a COD value of approximately 5 , the SNR is estimated to be $5000: 1$ for $1 \mathrm{~Hz}$ reports.

\section{Data availability}

AERONET cloud-mode data from the DOE ARM TCAP campaign (ARM_Highlands_MA site, 2013) are available on a restricted basis, due to the research and development phase characterizing AERONET cloud mode, from http://aeronet. gsfc.nasa.gov/cgi-bin/type_piece_of_map_cloud. For similar reasons, TWST data from TCAP are not presently available from the ARM data archive.

Acknowledgements. The authors thank Christine Chiu of the University of Reading, UK, for help in understanding the AERONET cloud-mode algorithm, Brent Holben and the AERONET team at NASA Goddard, Laurie Gregory and Richard Wagener of Brookhaven National Lab, and Ilya Slutsker of Sigma Space Corp. for details on the particular AERONET sensor used at TCAP, as well as the TCAP PI Larry Berg of PNNL and Paul Ortega of LANL for permission to participate in TCAP, MAGIC PI Ernie Lewis of Brookhaven National Lab for the idea of a TWST deployment at TCAP, and Vaughan Ivens for invaluable assistance on site at Cape Cod. Data were obtained from the Atmospheric Radiation Measurement (ARM) Climate Research Facility sponsored by the US Department of Energy, Office of Science, Office of Biological and Environmental Research, Climate and Environment Sciences Division. The Cimel Sun-photometer data were collected by the US Department of Energy as part of the ARM Climate Research Facility and processed by the National Aeronautics and Space Administration's Aerosol Robotic Network (AERONET).

Edited by: A. Sayer

Reviewed by: three anonymous referees

\section{References}

Baldridge, A. M., Hook, S. J., Grove, C. I., and Rivera, G.: The ASTER Spectral Library Version 2.0., Remote Sens. Environ., 113, 711-715, 2009.

Barker, H. W. and Marshak, A.: Inferring optical depth of broken clouds above green vegetation using surface solar radiometric measurements, J. Atmos. Sci., 58, 2989-3006, 2001.

Berk, A., Anderson, G. P., Acharya, P. K., Bernstein, L. S., Muratov, L., Lee, J., Fox, M., Adler-Golden, S. M., Chetwynd, J. H., Hoke, M. L., Lockwood, R. B., Gardner, J. A., Cooley, T. W., Borel, C. C., Lewis, P. E., and Shettle, E. P.: MODTRAN5: 2006 update, P. Soc. Photo-Opt. Ins., 6233, 62331F, 2006. 
Chiu, J. C., Marshak, A., Knyazikhin, Y., Wiscombe, W., Barker, H., Barnard, J. C., and Luo, Y.: Remote sensing of cloud properties using ground-based measurements of zenith radiance, J. Geophys. Res., 111, D162011, doi:10.1029/2005JD006843, 2006.

Chiu, J. C., Huang, C.-H., Marshak, A., Slutsker, I., Giles, D. M., Holben, B. N., Knyazikhin, Y., and Wiscombe, W. J.: Cloud optical depth retrievals from the Aerosol Robotic Network (AERONET) cloud mode operation, J. Geophy. Res., 115, D14202, doi:10.1029/2009JD013121, 2010.

Chiu, J. C., Marshak, A., Huang, C.-H., Várnai, T., Hogan, R. J., Giles, D. M., Holben, B. N., O'Connor, E. J., Knyazikhin, Y., and Wiscombe, W. J.: Cloud droplet size and liquid water path retrievals from zenith radiance measurements: examples from the Atmospheric Radiation Measurement Program and the Aerosol Robotic Network, Atmos. Chem. Phys., 12, 1031310329, doi:10.5194/acp-12-10313-2012, 2012.

Fielding, M. D., Chiu, J. C., Hogan, R. J., and Feingold, G.: A novel ensemble method for retrieving properties of warm cloud in 3-D using ground-based scanning radar and zenith radiances, J. Geophys. Res. Atmos., 119, 10912-10930, doi:10.1002/2014JD021742, 2014.

Harrison, I. and Min, Q.-L.: Photon pathlength distributions in cloudy atmospheres from ground-based high-resolution $\mathrm{O}_{2}$ Aband spectroscopy. IRS'96 Current Problems in Atmospheric Radiation, edited by: Smith, W. L. and Stamnes, K., Deepak Publishers, 594-597, 1997.

Kikuchi, N., Nakjima, T., Kumagai, H., Kuroiwa, H., Kamei, A., Nakamura, R., and Nakajima, T. Y.: Cloud optical thickness and effective particle radius derived from transmitted solar radiation measurements: Comparison with cloud radar observations, J. Geophys. Res., 111, D07205, doi:10.1029/2005JD006363, 2006.

King, M. D.: Determination of the scaled optical thickness of clouds from reflected solar radiation measurements, J. Atmos. Sci., 44, 1734-1751, 1987.

Liu, J., Li, Z., Zheng, Y., Chiu, J. C., Zhao, F., Cadeddu, M., Weng, F., and Cribb, M.: Cloud optical and microphysical properties derived from ground-based and satellite sensors over a site in the Yangtze Delta region, J. Geophys. Res.-Atmos., 118, 9141-9152, doi:10.1002/jgrd.50648, 2013.

Marshak, A., Knyazikhin, Y., Davis, A., Wiscombe, W., and Pilewskie P.: Cloud-vegetation interaction. Use of normalized difference cloud index for estimation of cloud optical thickness, Geophys. Res. Lett., 27, 1695-1698, 2000.

Marshak, A., Knyazikhin, Y., Evans, K. D., and Wiscombe, W. J.: The "RED versus NIR" plane to retrieve broken-cloud optical depth from ground-based measurements, J. Atmos. Sci., 61, 1911-1925, 2004.
McBride, P. J., Schmidt, K. S., Pilewskie, P., Kittelman, A. S., and Wolfe, D. E.: A spectral method for retrieving cloud optical thickness and effective radius from surface-based transmittance measurements, Atmos. Chem. Phys., 11, 7235-7252, doi:10.5194/acp-11-7235-2011, 2011.

Melnikova, I., Domnin, P., Mikhailov, V., and Radionov, V.: Optical cloud characteristics derived from measurements of reflected or transmitted solar radiation, J. Atmos. Sci., 57, 2135-2143, 2000.

Mulliken, R. S.: Interpretation of the atmospheric oxygen bands; electronic levels of the oxygen molecule, Nature, 122, 505, doi:10.1038/122505a0, 1928.

Nakajima, T. and King, M.: Determination of the optical thickness and effective particle radius of clouds from reflected solar radiation measurements. Part I: Theory, J. Atmos. Sci., 47, 18781893, 1990.

NASA Goddard Space Flight Center: Aerosol Robotic Network (AERONET) - Cloud Mode Data Display Interface, available at: http://aeronet.gsfc.nasa.gov/cgi-bin/type_piece_of_ map_cloud, 29 August 2016.

Pfeilsticker, K., Erle, F., Funk, O., Veitel, H., and Platt, U.: Cloudy sky photon pathlength as derived from DOAS-observations, IRS'96 Conference, Fairbanks, AK, 19-24 August 1996.

Pfeilsticker, K., Erle, F., Funk, O., Veitel, H., and Platt, U.: First geometrical pathlengths probability density function derivation of the skylight from spectroscopically highly resolving oxygen Aband observations, J. Geophys. Res., 103, 11483-11504, 1998.

Stamnes, K., Tsay, S.-C., Wiscombe, W., and Jayaweera, K.: Numerically stable algorithm for discrete-ordinate-method radiative transfer in multiple scattering and emitting layered media, Appl. Optics, 27, 2502-2509, doi:10.1364/AO.27.002502, 1988.

Stephens, G. L.: Radiation profiles in extended water clouds. II: Parameterization schemes, J. Atmos. Sci., 35, 2123-2132, doi:10.1175/1520-0469(1978)035<2123:RPIEWC>2.0.CO;2, 1978.

Wark, D. Q. and Mercer, D. M.: Absorption in the atmosphere by the oxygen A band, Appl. Optics, 4, 839-844, 1965.

Wood, R. and Hartmann, D. L.: Spatial variability of liquid water path in marine low cloud: The importance of mesoscale cellular convection, J. Climate, 19, 1748-1764, doi:10.1175/JCLI3702.1, 2006. 